\title{
Correction to: Non-Invasive Multimodality Imaging Directly Shows TRPM4 Inhibition Ameliorates Stroke Reperfusion Injury
}

\author{
Bo Chen ${ }^{1}$. Gandi $\mathrm{Ng}^{1} \cdot$ Yahui Gao ${ }^{1}$. See Wee Low ${ }^{1} \cdot$ Edwin Sandanaraj $^{1,2,3,4} \cdot$ Boominathan Ramasamy $^{5}$. \\ Sakthivel Sekar ${ }^{5}$. Kishore Bhakoo ${ }^{5}$. Tuck Wah Soong ${ }^{6,7}$ - Bernd Nilius ${ }^{8} \cdot$ Carol Tang $^{1,9,10}$ • Edward G. Robins ${ }^{5}$. \\ Julian Goggi ${ }^{5}$. Ping Liao ${ }^{1,9,11}$
}

Published online: 17 April 2019

(C) Springer Science+Business Media, LLC, part of Springer Nature 2019

\section{Correction to: Translational Stroke Research (2019) 10:91-103 https://doi.org/10.1007/s12975-018-0621-3}

The original version of the article unfortunately contained an error.

In Fig. 2a upper panel, in which the labeling for the middle picture should be TRPM4 (2hr) instead of TRPM4 (6hr).

The correct image is shown below:

The online version of the original article can be found at https://doi.org/ 10.1007/s12975-018-0621-3

Julian Goggi

julian_goggi@sbic.a-star.edu.sg

$\triangle$ Ping Liao

ping_liao@nni.com.sg

1 Department of Research, National Neuroscience Institute, Singapore, Singapore

2 School of Biological Sciences, Nanyang Technological University, Singapore, Singapore

3 Singapore Institute for Clinical Sciences, Singapore, Singapore

4 Singapore Bioimaging Consortium, Singapore, Singapore

5 Singapore Bioimaging Consortium, Agency for Science, Technology and Research (A*STAR), 07-10 HELIOS, 11 Biopolis Way, Singapore 138667, Singapore
6 Department of Physiology, Yong Loo Lin School of Medicine, National University of Singapore, Singapore, Singapore

7 Ion Channel Research Lab, Singapore, Singapore

8 Department of Cellular and Molecular Medicine, KU Leuven, Leuven, Belgium

9uke-National University of Singapore Graduate Medical School, Singapore, Singapore

10 National Cancer Centre, Singapore, Singapore

11 Calcium Signalling Lab, National Neuroscience Institute, 11 Jalan Tan Tock Seng, Singapore 308433, Singapore 
Fig. 2 TRPM4 expression in the hyperacute phase of stroke. a Immunofluorescent staining of cerebral blood vessels with antibodies against vWF and TRPM4 in the ipsilateral striatum $2 \mathrm{~h}$ (upper panel) and $6 \mathrm{~h}$ (lower panel) after stroke induction. Arrows: smaller vessels do not express TRPM4 at $2 \mathrm{~h}$ post-stroke induction. Scale bars: $50 \mu \mathrm{m}$. b Comparison of TRPM4-positive blood vessels in the ipsilateral hemispheres at 2 and $6 \mathrm{~h}$ poststroke. ( $n=6$ rats, $* * p=0.0109$, Student's $t$ test)
A
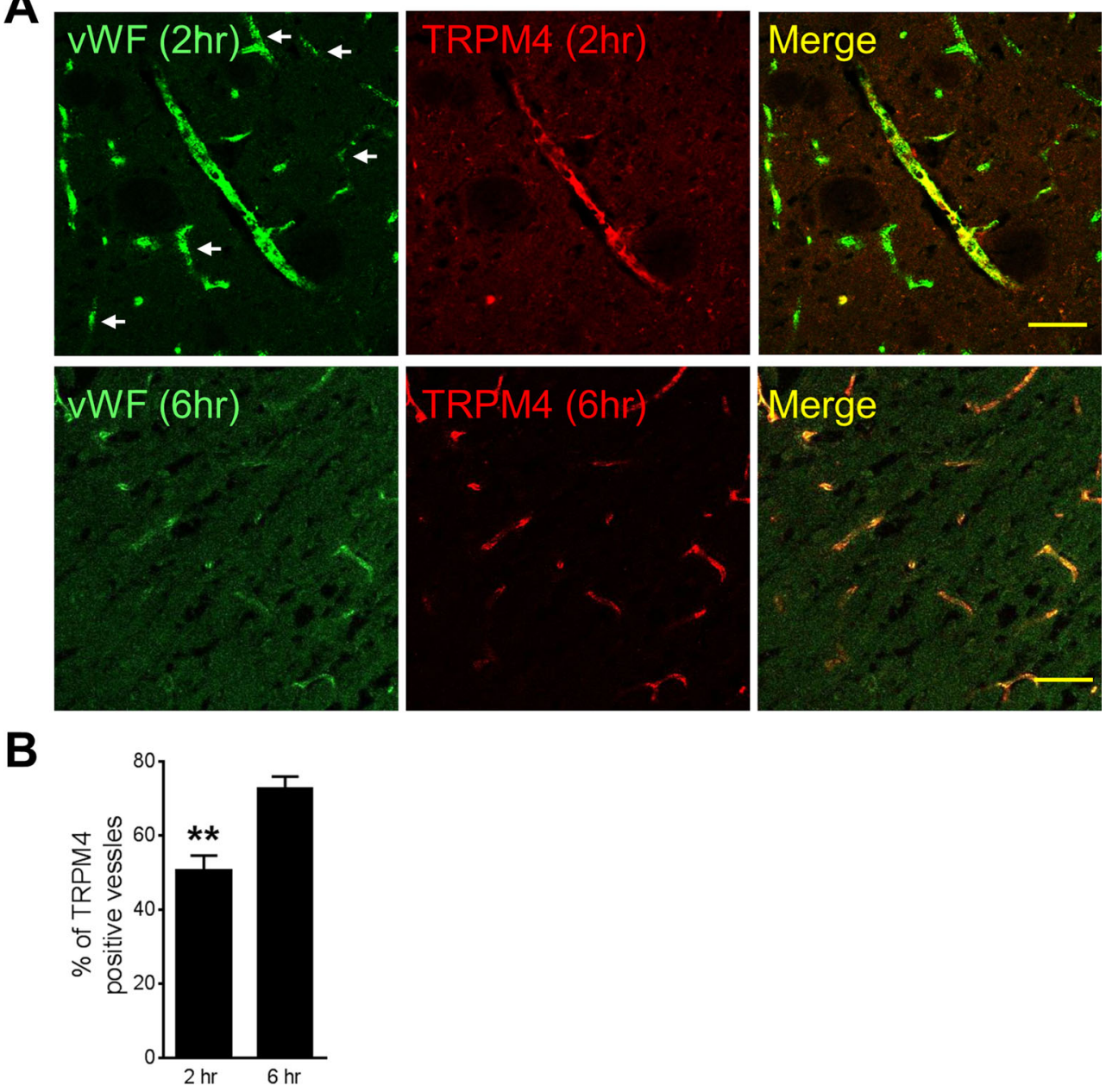

Publisher's Note Springer Nature remains neutral with regard to jurisdictional claims in published maps and institutional affiliations. 